\title{
'|||||||||||||||||||||||||||||||||||||||||||||||||||||||||||||||||||||||.
}

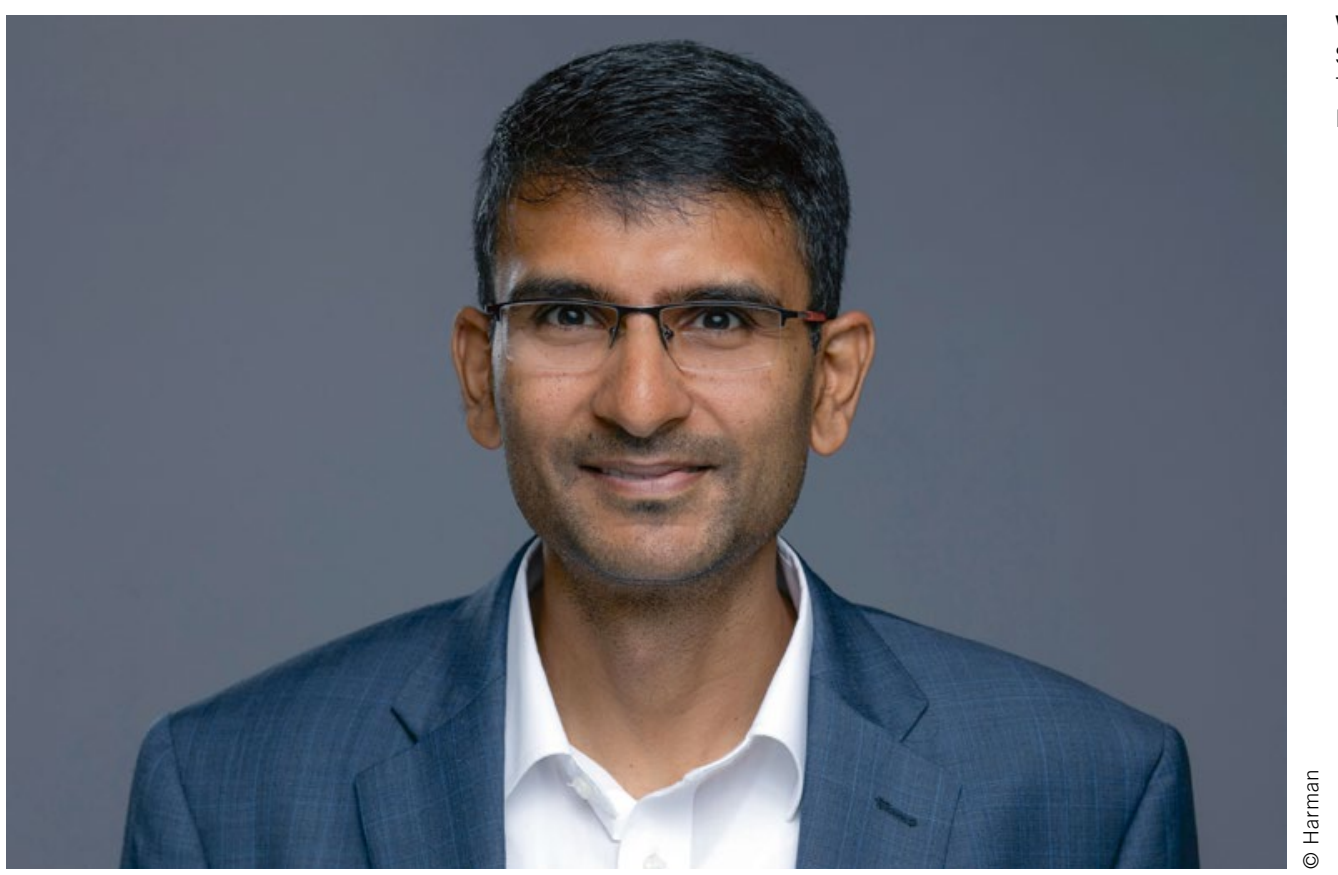

Vishnu Sundaram

Senior Vice President,

Telematics Business Unit der

Harman Automotive Division

\section{G, der Pkw und die Stadt}

Die Entwicklung von Fahrerassistenzsystemen ist zu einem der am meisten beachteten Trends in der Automobilindustrie geworden. Sowohl mit der zunehmenden Verbreitung des Mobilfunkstandards 5G als auch mit der einhergehenden Cellular-Vehicleto-Everything(C-V2X)-Vernetzung wird es neuen Technologien mit lebensrettendem Potenzial ermöglicht, rasch auf den Markt zu kommen. Diese C-V2X-Vernetzung gestattet es Automobilen, mit der städtischen Infrastruktur zu „sprechen“ und sicherere Interaktionen in urbanen Räumen zu ermöglichen.

Im Zuge dessen hat Harman eine neue 5G-fähige Lösung entwickelt, die als Vehicle-to-Pedestrian(V2P)-Technik bezeichnet wird. Sie nutzt 5G-Peer-to-Peer-Signale mit niedriger Latenzzeit und Proximity Scanning zur Identifikation und Benachrichtigung des Fahrers bei Objekten auf dem Fahrweg. In ähnlicher Weise erhalten Fußgänger oder Radfahrer mit einem C-V2Xfähigen Mobilgerät ebenfalls eine Warnung, wenn ein Fahrzeug ihren Weg kreuzt. Dadurch können Fahrer, Fußgänger und Radfahrer auf potenzielle Sicherheitsprobleme aufmerksam gemacht werden, selbst in Situationen, in denen Kameras keine Hindernisse erkennen können.

Die V2P-Technologie ist nur eine von vielen Sicherheitsverbesserungen, die durch C-V2X und eine situationsabhängige Erkennung zwischen 5G-fähigen Geräten ermöglicht werden. Im Zuge der Weiterentwicklung von 5G-Technologien und -Netzen schafft C-V2X neue Chancen für Fahrzeuge, mit ihrer Umgebung noch intensiver zu kommunizieren - einschließlich anderer Fahrzeuge, Straßeninfrastruktur und Kontrollsysteme sowie mit Verkehrsteilnehmern wie Fußgängern und Radfahrern, deren Sichtfeld eingeschränkt sein kann.

Der Übergang zu stärker vernetzten Straßen erfordert den Ausbau der derzeitigen Infrastruktur mit den notwendigen Hochgeschwindigkeitstechnologien. Die bestehende Infrastruktur kann dabei im wahrsten Sinne des Wortes als Datentreiber fungieren. So stattet Harman beispielsweise die bestehenden Stadtbusse in Seoul (Südkorea) mit Telematik-Kommunikationssystemen aus, die eine Echtzeit-Rückmeldung an einen zentralen Kontrollraum übermitteln. Dies ist nur ein Beispiel, wie Gemeinden damit beginnen können, eine ihrer wertvollsten Ressourcen - ihre Transportdaten - besser zu nutzen. Hierdurch können sie bessere Verkehrssysteme schaffen und schrittweise die Zukunft autonomer Fahrzeuge gestalten. Dieses Modell hat das Potenzial, sich in den Metropolen zu verbreiten, mit dem letztendlichen Ziel, die Zahl der Verkehrszwischenfälle so gering wie möglich zu halten.

Zurzeit konzentrieren wir uns auf die Sicherheits- und Fahrerinformationselemente der C-V2X-Technologie und stellen sicher, dass der Fahrer benachrichtigt wird und entsprechend reagieren kann. Diese Technologien werden zukünftig die Chance eröffnen, Fußgänger über einen bevorstehenden Zwischenfall zu informieren und korrigierend einzugreifen. Gemeinsam mit unserer Konzernmutter Samsung prüfen wir, wie diese Technologie zunächst mit Smartphones und später in diesem Jahrzehnt auch mit anderen 5G-fähigen Geräten wie Kopfhörern und Wearables kombiniert werden kann. 